\title{
On the existence of lateral waves
}

\author{
By
}

Mikio TsujI

(Communicated by Prof. S. Mizohata, Oct. 26, 1976)

\section{§1. Introduction}

We consider the reflection of the singularities at the boundary for hyperbolic equations with constant coefficients. In [5] and [6], we studied this problem in a very general framework, and determined the singular supports of the fundamental solutions by using the localization theorem. The wave which attracts our attension extremely is the lateral wave. Moreover there exists the case where it does not appear. The aim of this paper is to determine the case where the lateral wave arises.

In this paper we treat the following equation in the domain $\Omega=\{t>0$, $\left.x>0, y=\left(y_{1}, y_{2}, \cdots, y_{n-1}\right) \in R^{n-1}\right\}$ :

$$
\left\{\begin{array}{l}
P(D) u=\left(D_{t}^{2}-D_{x}^{2}-D_{y}^{2}\right)\left(a^{2} D_{t}^{2}-D_{x}^{2}-D_{y}^{2}\right) u=0 \quad \text { in } \Omega \\
\left(u, D_{t} u, D_{t}^{2} u, D_{t}^{3} u\right)=\left(0,0,0, i \delta_{(x-l, y)}\right) \text { on } \partial \Omega \cap\{t=0\}, \\
B_{j}\left(D_{t}, D_{x}, D_{y}\right) u=0 \quad \text { on } \partial \Omega \cap\{x=0\}, j=1,2,
\end{array}\right.
$$

where i) $a>1$ and $l>0$, ii) $D_{t}=-i \partial / \partial t, D_{x}=-i \partial / \partial x, D_{y_{i}}=-i \partial / \partial y_{i}$ and $D_{y}^{2}=$ $\sum_{i=1}^{n-1} D_{y_{i}}{ }^{2} . \quad B_{j}(j=1,2)$ are homogeneous differential operators of degree $m_{j}$ $\left(m_{1}<m_{2}\right)$ with constant coefficients. The waves governed by $P u=0$ propagate

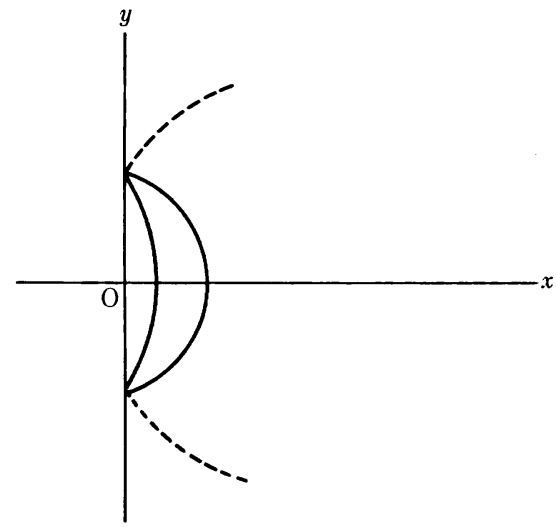

Figure 1

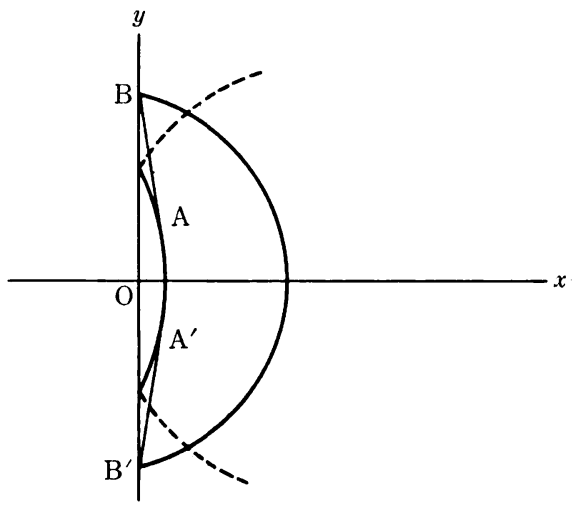

Figure 2 
by the speed 1 or $1 / a$. Hence, when an incident wave front surface impinges on the boundary, the number of the sheets of the reflected wave front surfaces is generally two. As showed in $\S 8$ of [2] and $\S 2.6$ of [6], the lateral wave appears when the wave of speed $1 / a$ impinges on the boundary. We show this phenomena by the following figures in the case where the boundary waves don't appear: Figure 1 and Figure 2 show the phenomena in $a^{2} l / \sqrt{a^{2}-1} \geqq t \geqq a l$ and $t>a^{2} l / \sqrt{a^{2}-1}$ respectively where the dotted lines show the incident wave front surface of speed $1 / a$ and the continuous lines show the reflected wave front surfaces. The outer semi-sheet in the figures is the reflected wave front surface of speed 1 and the inner one is of speed $1 / a$. The lines $A B$ and $A^{\prime} B^{\prime}$ are called as the lateral waves.

As Shirota showed in [4], in the case $B_{1}=1$ and $B_{2}=D_{x}^{2}$ the lateral wave does not appear, i.e., sing supp $u \nsupseteq\left\{A B, A^{\prime} B^{\prime}\right\}$. The reason is that in this case, although the reflected wave front surface of speed $1 / a$ appears, the reflected wave of speed 1 does not arise. But, even if two sheets of the reflected wave front surfaces appear, there exists the case where the lateral wave does not arise, for epample $B_{1}=1$ and $B_{2}=\left(D_{t}^{2}-D_{x}^{2}-D_{y}^{2}\right) D_{x}$. However, in this case, when the incident wave front of speed 1 hits on the boundary, the number of the reflected wave front surfaces is one, i.e., the reflected wave front of speed $1 / a$ does not appear. Taking care of the above facts, we get the following

Theorem 1. The necessary and sufficient condition that the lateral wave appears is that, whenever the incident wave front surface of speed 1 or 1/a impinges on the boundary, two sheets of the reflected wave front surfaces always appear.

We say this condition in other various ways, which are given in $\S 2$ and $\S 4$. In the following discussions we limit ourselves to give the sketch of the proofs, because the method of them is essentially given in [6].

\section{§2. Notations and Lemmas.}

We write the dual coordinates of $(t, x, y)$ by $(\sigma, \xi, \eta) \in R^{n+1}$, and put $\tau=\sigma-i \gamma(\gamma>0)$. We write $P_{+}(\tau, \eta ; \xi)=\left(\xi-\xi_{1}{ }^{+}(\tau, \eta)\right)\left(\xi-\xi_{2}{ }^{+}(\tau, \eta)\right)$ where $\xi_{1}{ }^{+}(\tau, \eta)=\sqrt{\tau^{2}-\eta^{2}}$ and $\xi_{2}{ }^{+}(\tau, \eta)=\sqrt{a^{2} \tau^{2}-\eta^{2}}\left(\operatorname{Im} \xi_{i}^{+}>0, i=1,2\right)$. We define the matrix $L(\tau, \eta)$ by

$$
L(\tau, \eta)=\left[\frac{1}{2 \pi i} \oint_{\Gamma_{+}} \frac{B_{j}(\tau, \xi, \eta) \xi^{j-1}}{P_{+}(\tau, \eta ; \xi)} d \xi\right]_{1 \leq i, j \leq 2}
$$

where $\Gamma_{+}$is a simple closed path containing $\xi_{i}{ }^{+}(\tau, \eta)(i=1,2)$. We put $R(\tau, \eta)$ $=\operatorname{det} L(\tau, \eta)$ and $R_{i j}(\tau, \eta)=(j, i)$-cofactor of $L(\tau, \eta)$. We assume

(A) The mixed problem (1.1) is E-well posed.

Then, as $R(\tau, \eta) \neq 0$ for $\tau=\sigma-i \gamma(\gamma>0)$ and $\eta \in R^{n-1}$, the solution $u$ of (1.1) is given by $u=E_{0}(t, x, y ; l)+E_{1}(t, x, y ; l)$ where 


$$
\begin{aligned}
& E_{0}(t, x, y ; l)=\left(\frac{1}{2 \pi}\right)^{n+1} \int_{R^{n+1}} \frac{e^{i\left(t^{\tau}+(x-l) \xi+y \eta\right)}}{P(\tau, \xi, \eta)} d \sigma d \xi d \eta, \\
& E_{1}(t, x, y ; l)=\sum_{i, j=1}^{2}\left(\frac{1}{2 \pi}\right)^{n+2} \int_{R^{n+2}} \frac{R_{i j}(\tau, \eta) \xi^{i-1} B_{j}(\tau, \zeta, \eta)}{R(\tau, \eta) P+(\tau, \eta ; \xi) P(\tau, \zeta, \eta)} \\
& \quad \times e^{i(t+x \xi+y \eta-l \zeta)} d \sigma d \xi d \eta d \zeta .
\end{aligned}
$$

Our aim is to determine the singular support of $E_{1}$, following the method given in Chapter 2 of [6]. We proved there that sing supp $E_{1}$ generally consists of the ordinary reflected wave front surfaces, the lateral waves and the boundary waves, and that it has no other singularity. The boundary waves are the singularity contributed by real zeros of $R(\tau, \eta)$. But, as we are concerned with only the lateral wave, we shall have no discussion about the boundary waves. By the simple calculation we have

$$
\begin{aligned}
E_{1}(t, x, y ; l) & =\sum_{i=1}^{2}\left(\frac{1}{2 \pi}\right)^{n+2} \int_{R^{n+2}} \frac{H_{i}(\tau, \zeta, \eta) e^{i\left(t^{\tau}+x \xi+y \eta-l \zeta\right)}}{R^{\prime}(\tau, \eta)\left(\xi-\xi_{i}^{+}(\tau, \eta)\right) P(\tau, \zeta, \eta)} d \sigma d \xi d \eta d \zeta \\
& \equiv \sum_{i=1}^{2} E_{1}^{(i)}(t, x, y ; l)
\end{aligned}
$$

where, by putting $B_{i}(\xi)=B_{i}(\tau, \xi, \eta), R^{\prime}(\tau, \eta)=B_{1}\left(\xi_{1}^{+}\right) B_{2}\left(\xi_{2}^{+}\right)-B_{1}\left(\xi_{2}{ }^{+}\right) B_{2}\left(\xi_{1}{ }^{+}\right)$, $H_{1}(\tau, \zeta, \eta)=B_{1}(\zeta) B_{2}\left(\xi_{2}{ }^{+}\right)-B_{1}\left(\xi_{2}^{+}\right) B_{2}(\zeta)$ and $H_{2}(\tau, \zeta, \eta)=B_{1}(\zeta) B_{2}\left(\xi_{1}{ }^{+}\right)-$ $B_{1}\left(\xi_{1}^{+}\right) B_{2}(\zeta)$.

We consider the behavior of the reflected wave front surfaces when the incident wave of speed $1 / a$ touches the boundary. For this we localize $E_{1}$ at $P_{0}=\left(\sigma^{0}, \xi^{0}, \eta^{0}, \zeta^{0}\right)=\left(1, \xi_{i}{ }^{0}, \eta^{0}, \zeta^{0}\right)(i=1,2)$ where $\left(\eta^{0}\right)^{2}+\left(\zeta^{0}\right)^{2}=a^{2}, \zeta^{0}>0, \xi_{1}{ }^{0}=$ $+\sqrt{1-\left(\eta^{\circ}\right)^{2}}=-\sqrt{1-\left(\eta^{\circ}\right)^{2}}$ and $\xi_{2}^{0}=\sqrt{a^{2}-\left(\eta^{\circ}\right)^{2}}=-\sqrt{a^{2}-\left(\eta^{\circ}\right)^{2}}$. We put

$$
K_{j}(t, x, y ; l)=\left(\frac{1}{2 \pi}\right)^{n+2} \int_{R^{n+2}} \frac{H_{j}\left(\tau,-\xi_{j}^{+}, \eta\right) e^{i(t \tau+x \xi+y \eta-l \zeta)}}{R^{\prime}(\tau, \eta) \cdot\left(\xi-\xi_{j}^{+}(\tau, \eta)\right) \cdot P(\tau, \zeta, \eta)} d \sigma d \xi d \eta d \zeta
$$

for $j=1$ and 2. As $\left(H_{j}(\tau, \zeta, \eta)-H_{j}\left(\tau,-\xi_{2}{ }^{+}, \eta\right)\right) / P(\tau, \zeta, \eta)$ is analytic at $(\tau, \zeta, \eta)=\left(1, \zeta^{0}, \eta^{0}\right)$ with respect to $\zeta$, we get the following

Lemma 2. For any $p>0$ and the above $P_{0}$, it follows

$$
s^{p} e^{-i s\left(t^{0}+x \xi^{0}+y \eta^{0}-l \zeta^{0}\right)}\left(E_{1}^{(j)}(t, x, y ; l)-K_{j}(t, x, y ; l)\right) \longrightarrow 0, j=1,2,
$$

in $\mathscr{D}^{\prime}\left(\Omega \times R_{+}{ }^{1}\right)$ when $s \rightarrow \infty$.

Therefore we study the singularity of $K_{j}(j=1,2)$. At first we determine the singular support of $K_{1}$ by using the localization theorem given as Theorem 2.1 and 2.2 in [6]. We define

$$
R_{\left(\sigma^{0}, \eta^{0}\right)}(\tau, \eta)=\lim _{s \rightarrow \infty} s^{\rho_{0}} R\left(\left(\sigma^{0}, \eta^{0}\right)+s^{-1}(\tau, \eta)\right) \not \equiv 0,
$$

then $R_{\left(\sigma^{0}, \eta^{0}\right)}(\tau, \eta) \neq 0$ for $\tau=\sigma-i \gamma(\gamma>0)$ and $\eta \in R^{n-1}$. Assume $H_{1}\left(\tau,-\xi_{2}{ }^{+}, \eta\right) \not \equiv$ 0 . We expand $\exp \left(-i s\left(t+x \xi_{1}{ }^{0}+y \eta^{0}-l \zeta^{\circ}\right)\right) K_{1}$ asymptotically with respect to $s$ :

$$
e^{-i s\left(t+x \xi_{1}{ }^{0}+y \eta \eta^{0}-l \zeta^{0}\right)} K_{1} \sim \sum_{k=0}^{\infty} F_{k}{ }^{(1)}(t, x, y: l)(1 / s)^{e_{k}}
$$

where $e_{0}<e_{1}<e_{2} \cdots<e_{n} \rightarrow \infty$. Then, sing supp $K_{1} \supset \cup_{k=0}^{\infty} \operatorname{supp} F_{k}^{(1)}$, and 


$$
F_{0}{ }^{(1)}=\mathrm{const}\left(\frac{1}{2 \pi}\right)^{n+2} \int_{R^{n+2}} \frac{h_{1}(\tau, \eta) e^{i\left(t+x+x_{\xi}+y \eta-l \zeta\right)}}{R_{\left(1, \eta^{0}\right)}(\tau, \eta)\left(\tau-\xi_{1}{ }^{0} \xi-\eta^{0} \eta\right)\left(a^{2} \tau-\zeta^{0} \zeta-\eta^{0} \eta\right)} d \sigma d \xi d \eta d \zeta
$$

where $h_{1}$ is polynomial of $\tau$ and $\eta$.

Lemma 3. Let $Q\left(D_{t}, D_{x}, D_{y}\right)$ be a differential operator and

$$
T=(2 \pi)^{-n-1} \int_{R^{n+1}} \frac{e^{i\left(t^{\tau}+x \xi+y \eta\right)}}{\tau-a \xi-b \eta} d \sigma d \xi d \eta=i H(\dot{t}) \delta_{(x+a t, y+b t)} .
$$

If $\operatorname{supp} Q T \cong \operatorname{supp} T$, then $Q(a \xi+b \eta, \xi, \eta) \equiv 0$.

In this case, as $\xi_{1}{ }^{0} \neq 0, h_{1}\left(\xi_{1}{ }^{0} \xi+\eta^{0} \eta, \eta\right) \not \equiv$. Hence supp $F_{0}{ }^{(1)} \supset\{(t, x, y, l)$; $\left.t>0, x>0, l>0,\left(x, y+\eta^{0} l / \zeta^{0}\right)=-\left(t-a^{2} l / \zeta^{0}\right)\left(\xi_{1}{ }^{0}, \eta^{0}\right)\right\}$, which means that the reflected wave of speed 1 appears. By the same discussions for $K_{2}$, we see that, if $H_{2}\left(\tau,-\xi_{2}{ }^{+}, \eta\right) \not \equiv 0$, the reflected wave of speed $1 / a$ arises.

Next we consider $K_{2}$ at $\left|\eta^{0}\right|=1$. We represent as

$$
\frac{H_{2}\left(\tau,-\xi_{2}{ }^{+}, \eta\right)}{R(\tau, \eta)}=\frac{a_{2}(\tau, \eta)}{a_{1}(\tau, \eta)}+\frac{a_{4}(\tau, \eta)}{a_{3}(\tau, \eta)} \xi_{1}{ }^{+}(\tau, \eta)
$$

where $a_{i}(\tau, \eta)$ are analytic at $\left(1, \eta^{0}\right)$. Assume $a_{4}(\tau, \eta) / a_{3}(\tau, \eta) \equiv 0$. We expand $\exp \left\{-i s\left(t+x \xi_{2}{ }^{0}+y \eta^{0}-l \zeta^{0}\right)\right\} K_{2}$ asymptotically with respect to $s$, then there exists the term such that

$$
F_{j}^{(2)}=\left(\frac{1}{2 \pi}\right)^{n+2} \int_{R^{n+2}} \frac{h_{2}(\tau, \eta)^{+} \sqrt{\tau-\eta^{0} \eta} e^{i\left(t^{\tau}+x \xi+y \eta-l \zeta\right)}}{h_{3}\left(a^{2} \tau-\xi_{2} \xi \xi-\eta^{0} \eta\right)\left(a^{2} \tau-\zeta^{0} \zeta-\eta^{0} \eta\right)} d \sigma d \xi d \eta d \zeta
$$

where $h_{i}(\tau, \eta)(i=2,3)$ are polynomial of $\tau$ and $\eta$.

Lemma 4. Let $Q\left(D_{t}, D_{y}\right)$ be any differential operator with respect to $t$ and $y$, and put

$$
T=\left(\frac{1}{2 \pi}\right)^{n+1} \int_{R^{n+1}} \frac{+\sqrt{\tau+a \eta}}{\tau+b \xi+c \eta} e^{i\left(t^{\tau}+x \xi+y \eta\right)} d \sigma d \xi d \eta
$$

where $b \neq 0$. Then supp $Q T=\operatorname{supp} T$.

Proof. We change the transformation of the variables $(t, x, y) \rightarrow(p, q, r)$ as

$$
(t, x, y)=p \vec{n}_{0}+q \vec{n}_{1}+\sum_{i=2}^{n} r_{i} \vec{n}_{i}, r=\left(r_{2}, \cdots, r_{n}\right),
$$

where $\vec{n}_{0}=(1,0, a) / \sqrt{1+a^{2}}, \vec{n}_{1}=(1, b, c) / \sqrt{1+b^{2}+c^{2}}$ and $\left\{\vec{n}_{i}\right\}_{i=0,1, \cdots, n}$ is the orthogonal basis of $R^{n+1}$. Then, using Lemma 2.12 in [6], we get

$$
T=\text { const } p_{+}{ }^{-3 / 2} H_{s}(p, q) \otimes \delta_{r}
$$

where $S=\{(p, q) ; p>0, q>0\}$ and

$$
H_{s}(p, q)= \begin{cases}1, & (p, q) \in S \\ 0, & (p, q) \notin S .\end{cases}
$$

We define $\tilde{Q}$ by $\widetilde{Q}\left(D_{p}, D_{q}, D_{r}\right)=Q\left(D_{t}, D_{y}\right)$. As $Q$ does not contain $D_{x}$, $\widetilde{Q}$ contains the term $D_{p}^{i} D_{r}^{j}$, i.e., $\widetilde{Q}$ is not represented as $\widetilde{Q}=Q^{*}(D) D_{q}$. Since 
$T$ contains the fractional power of $p$, supp $Q T=\operatorname{supp} T$.

Q.E.D.

Therefore, if $a_{4} / a_{3} \not \equiv 0, \operatorname{supp} F_{j}^{(2)} \supset\left\{(t, x, y, l) ; t>0, l>0,\left(x, y+\eta^{0} l / \zeta^{0}\right)=\right.$ $\left.-\left(t-a^{2} l / \zeta^{0}\right)\left\{v\left(0, \eta^{0}\right)+(1-v)\left(\xi_{2}{ }^{0}, \eta^{0}\right) / a^{2}\right\}, 0 \leqq v \leqq 1\right\}$, which means the existence of the lateral wave. We see easily that $a_{4} / a_{3} \not \equiv 0$ is equivalent to the condition that i) $H_{i}\left(\tau,-\xi_{2}{ }^{+}(\tau, \eta), \eta\right) \not \equiv 0(i=1,2)$ and ii) $B_{2}\left(\xi_{1}{ }^{+}\right) / B_{1}\left(\xi_{1}{ }^{+}\right)$is not meromorphic. Hence, if the lateral wave appears, two sheets of the reflected wave front surfaces arise. Combining the above results, we get the following

Theorem 5. The necessary and sufficient condition for (1.1) to have the lateral waves is that i) $H_{i}\left(\tau,-\xi_{2}^{+}(\tau, \eta), \eta\right) \not \equiv 0(i=1,2)$ and $\left.i i\right) B_{2}\left(\xi_{1}{ }^{+}\right) / B_{1}\left(\xi_{1}{ }^{+}\right)$ is not meromorphic.

\section{§. Proof of Theorem 1 .}

The necessary and sufficient condition for $B_{2}\left(\xi_{1}^{+}\right) / B_{1}\left(\xi_{1}^{+}\right)$to be meromorphic is that $B_{i}(i=1,2)$ are represented as follows:

$$
\left\{\begin{array}{l}
B_{1}(\tau, \xi, \eta)=q_{1}(\tau, \xi, \eta)\left(\tau^{2}-\xi^{2}-\eta^{2}\right)+c_{1}(\tau, \eta)\left(d_{0}(\tau, \eta) \xi+d_{1}(\tau, \eta)\right), \\
B_{2}(\tau, \xi, \eta)=q_{2}(\tau, \xi, \eta)\left(\tau^{2}-\xi^{2}-\eta^{2}\right)+c_{2}(\tau, \eta)\left(d_{0}(\tau, \eta) \xi+d_{1}(\tau, \eta)\right)
\end{array}\right.
$$

where i) $q_{i}$ are polynomial of $(\tau, \xi, \eta)$ and ii) $c_{i}(i=1,2)$ and $d_{i}(i=0,1)$ are polynomial of $(\tau, \eta)$. By the same discussions as in $\S 2$, we get

Lemma 6. Assume that, when the incident wave front surface of speed 1 impinges on the boundary, two reflected wave front surfaces appear. Then

$$
H_{i}\left(\tau,-\xi_{1}^{+}(\tau, \eta), \eta\right) \not \equiv 0, i=1,2 .
$$

Moreover the convers is true.

If $B_{i}(i=1,2)$ are represented as $(3.1), H_{2}\left(\tau,-\xi_{1}{ }^{+}(\tau, \eta), \eta\right) \equiv 0$, which means that the reflected wave of speed $1 / a$ does not appear. Therefore, if two sheets of the reflected wave front surfaces appear in this case, $B_{2}\left(\xi_{1}^{+}\right) / B_{1}\left(\xi_{1}^{+}\right)$ is not meromorphic. We see easily that the converse is true. From these facts, we get Theorem 1 stated in $§ l$.

\section{\$4. Remarks on the condition (ii) of Theorem 5 .}

Let $B_{2}\left(\xi_{1}^{+}\right) / B_{1}\left(\xi_{1}^{+}\right)$be meromorphic, that is to say, (3.1) be satisfied. $E_{1}$ defined by $(2.2)$ is the solution of the following equation:

$$
\left\{\begin{array}{l}
P(D) E_{1}=0 \quad \text { in } D=\left\{(t, y) \in R^{n}, x>0\right\}, \\
B_{j}(D) E_{1}=g_{j} \text { on } \partial D=\left\{(t, y) \in R^{n}, x=0\right\}, j=1,2,
\end{array}\right.
$$

where

$$
g_{j}(t, y ; l)=-\left(\frac{1}{2 \pi}\right)^{n+1} \int_{R^{n+1}} \frac{B_{j}(\tau, \xi, \eta)}{P(\tau, \xi, \eta)} e^{\imath\left(t^{\tau}-l \xi+y \eta\right)} d \sigma d \xi d \eta .
$$

We put $v=\left(D_{t}^{2}-D_{x}^{2}-D_{y}^{2}\right) E_{1}$, then

$$
\left\{\begin{array}{l}
\left(a^{2} D_{t}^{2}-D_{x}^{2}-D_{y}^{2}\right) v=0 \text { in } D, \\
B_{1}{ }^{\prime}\left(D_{t}, D_{x}, D_{y}\right) v=g_{1}{ }^{\prime}(t, y ; l) \text { on } \partial D,
\end{array}\right.
$$


where $B_{1}{ }^{\prime}=c_{1}\left(D_{t}, D_{y}\right) q_{2}\left(D_{t}, D_{x}, D_{y}\right)-c_{2}\left(D_{t}, D_{y}\right) q_{1}\left(D_{t}, D_{x}, D_{y}\right)$ and $g_{1}{ }^{\prime}=c_{1}\left(D_{t}\right.$, $\left.D_{y}\right) g_{2}(t, y ; l)-c_{2}\left(D_{t}, D_{y}\right) g_{1}(t, y ; l)$. Taking care of $(3.1)$, we see

$$
g_{1}^{\prime}(t, y ; l)=-\left(\frac{1}{2 \pi}\right)^{n+1} \int_{R^{n+1}} \frac{B_{1}^{\prime}(\tau, \xi, \eta)}{a^{2} \tau^{2}-\xi^{2}-\eta^{2}} e^{i(t \tau-l \xi+y \eta)} d \sigma d \xi d \eta
$$

$E_{1}(t, x, y ; l)$ satisfies the following equation:

$$
\left\{\begin{array}{l}
\left(D_{t}^{2}-D_{x}{ }^{2}-D_{y}{ }^{2}\right) E_{1}=v \quad \text { in } D, \\
B_{2}{ }^{\prime}\left(D_{t}, D_{x}, D_{y}\right) E=g_{2}{ }^{\prime}(t, y ; l) \text { on } \partial D,
\end{array}\right.
$$

where $B_{2}{ }^{\prime}=d_{0}\left(D_{t}, D_{y}\right) D_{x}+d_{1}\left(D_{t}, D_{y}\right)$ and $g_{2}{ }^{\prime}$ is expressed as

$$
\begin{aligned}
& g_{2}{ }^{\prime}(t, y ; l)=-\left(\frac{1}{2 \pi}\right)^{n+1} \int_{R^{n+1}} \frac{B_{2}{ }^{\prime}(\tau, \xi, \eta)}{P(\tau, \xi, \eta)} e^{i\left(t^{\tau}-l \xi+y \eta\right)} d \sigma d \xi d \eta \\
& -\left(\frac{1}{2 \pi}\right)^{n+1} \int_{R^{n+1}} \frac{q_{2}\left(\tau, \xi_{2}{ }^{+}, \eta\right) q_{1}(\tau, \xi, \eta)-q_{1}\left(\tau, \xi_{2}{ }^{+}, \eta\right) q_{2}(\tau, \xi, \eta)}{B_{1}{ }^{\prime}\left(\tau, \xi_{2}{ }^{+}(\tau, \eta), \eta\right) \cdot\left(a^{2} \tau^{2}-\xi^{2}-\eta^{2}\right)} e^{\imath\left(t^{\tau}-l \xi+y \eta\right)} d \sigma d \xi d \eta .
\end{aligned}
$$

In this case Lopatinski's determinant is

$$
R(\tau, \eta)=\left(\xi_{1}{ }^{+}-\xi_{2}{ }^{+}\right)^{-1}\left(1-a^{2}\right) \tau^{2} \cdot B_{1}{ }^{\prime}\left(\tau, \xi_{2}{ }^{+}(\tau, \eta), \eta\right) \cdot B_{2}{ }^{\prime}\left(\tau, \xi_{1}{ }^{+}(\tau, \eta), \eta\right) .
$$

As $(1.1)$ is $\mathcal{E}$-well posed, $R(\tau, \eta)$ must be hyperbolic, i.e., $R(1,0) \neq 0$ and $R(\tau, \eta) \neq 0$ for $\tau=\sigma-i \gamma(\gamma>0)$ and $\eta \in R^{n-1}$, which means the hyperbolicity of $B_{1}{ }^{\prime}\left(\tau, \xi_{2}{ }^{+}(\tau, \eta), \eta\right)$ and $B_{2}{ }^{\prime}\left(\tau, \xi_{1}{ }^{+}(\tau, \eta), \eta\right)$. Hence (4.3) and (4.4) are $\mathcal{E}$-well posed and (4.5) is well defined. From the above results we have the following

Theorem 7. If the condition (ii) in Theorem 5 is not satisfied, the mixed problem (1.1) is decomposed into the iteration of two mixed problems (4.3) and (4.4) which are E-well posed.

Example. Let $m_{1} \leqq 1$ and $m_{2}$ be any positive integer in the above case. Then $B_{2}{ }^{\prime}\left(D_{t}, D_{x}, D_{y}\right)=B_{1}\left(D_{t}, D_{x}, D_{y}\right)$ and $g_{2}{ }^{\prime}(t, y ; l)=g_{1}(t, y ; l)$ defined by (4.2). Hence (4.3) and (4.4) are very simple.

In the mixed problem for wave equation, the lateral wave does not appear. Therefore, it is reasonable that, if the lateral wave appears, (1.1) can not be decomposed into two mixed problems as (4.3) and (4.4).

\section{Department of Mathematics Kyoto SANGYo UniverSITY 603 KYOTO, JAPAN.}

\section{References}

[1] Atiyah-Bott-Garding, Lacunas for hyperbolic differential operators with constant coefficients. I. Acta Math., 124, 109-198 (1970).

[2] G. F. D. Duff, On the wave fronts, and boundary waves. Comm. Pure Appl. Math., 17, 189-225 (1964).

[ 3 ] R. Sakamoto, E-well posedness for hyperbolic mixed problems with constant coefficients. J. Math. Kyoto Univ., 14, 93-118 (1974). 
[4] T. Shirota, Propagation of smoothness for hyperbolic mixed problems with constant coefficients. Proc. of a conference at Katata, 1972 (in japanese).

[5] M. Tsuji, Fundamental solutions of mixed problems for hyperbolic equations with constant coefficients. Proc. Japan Acad., 51, 369-373 (1975).

[6] M. Tsuji, Propagation of the singularities for hyperbolic equations with constant coefficients. Jap. J. Math., New Series, 2 (No. 2), 361-410 (1976). 